Pacific Journal of Mathematics

FRACTIONAL POWERS OF CLOSED OPERATORS AND THE
SEMIGROUPS GENERATED BY THEM 


\section{FRACTIONAL POWERS OF CLOSED OPERATORS AND THE SEMIGROUPS GENERATED BY THEM}

\section{A. V. BALAKRISHNAN}

Fractional powers of closed linear operators were first constructed by Bochner [2] and subsequently Feller [3], for the Laplacian operator. These constructions depend in an essential way on the fact that the Laplacian generates a semigroup. Phillips [6] in fact showed that these constructions (for positive indices less than one) were part of a more general one based on the Kolmogoroff-Levy representation theorem for infinitely divisible distributions. Finally, the present author constructed an operational calculus [1] for infinitesimal generators affording in particular a systematic study of the representation and properties of these operators.

In this paper we obtain a new construction for fractional powers in which it is not required that the base operator generate a semigroup; indeed its domain need not even be dense. Since the semigroup is not available, the previous constructions, based as they are on the RiemannLiouville integrals, are not possible. However, we shall show, if the resolvent exists for $\lambda>0$, and is $O(1 / \lambda$ ) for all $\lambda$, (a weaker condition will suffice at the origin, see $\S 7$ ), then fractional powers may still be constructed, using an abstract version of the Stieltjes transform.

It is immediate that a closed operator $A$, for which $\|\lambda R(\lambda, A)\|<$ $M$, does not necessarily generate a semigroup of any type. For a simple example, let the Banach space be $l_{2}(-\infty, \infty)$ and let $A$ correspond to multiplying the $n$th coordinate by $n(1+i)$ say. Then for $\lambda>0$, $\|R(\lambda, A)\| \leq \sqrt{2} / \lambda$, whereas $A$ does not generate a semigroup, since no right-half plane is free of the spectra of $A$. An example in which $A$ has no spectra in the right half plane and yet no semigroup is generated is given by Phillips [4].

The main motivation for the construction of fractional powers is the application to abstract Cauchy problems of the type:

$$
\frac{d^{n}}{d t^{n}} u(t) \pm A u(t)=0
$$

for $n \geq 2$, and it turns out that for the solution of (1.1), $A$ itself need not be an infinitesimal generator. In this paper we study only the case $n=2$, and we expect to consider the general case later.

The properties of newly constructed fractional powers are identical with those obtained in [1] for the case where $A$ is a generator, with one important difference; namely that $-(-A)^{\alpha}$ generates semigroups in 
general only for $\alpha \leq 1 / 2$. On the other hand, these are the only exponents that matter in the application to Cauchy problems of the type (1.1).

1. Construction of fractional powers. Let $A$ be a closed linear operator with domain and range in a Banach space $X$. Let each $\lambda>0$ belong to its resolvent set and let $\left(H_{0}\right)$

$$
\|\lambda R(\lambda, A)\|<M<\infty, \quad \lambda>0 .
$$

We have already noted that these conditions do not imply that $A$ generate a semigroup of any kind. Let $x \in D(A)$. Then for $0<\alpha<1$, the integral

$$
\int_{0}^{\infty} \lambda^{\alpha-1} R(\lambda, A) A x d \lambda
$$

where $\lambda^{\alpha}$ is taken positive, is convergent in the Bochner or absolute sense, since it can be expressed as

$$
\int_{0}^{1} \lambda^{\alpha-1}[R(\lambda, A) x-x] d \lambda+\int_{1}^{\infty} \lambda^{\alpha-1} R(\lambda, A) A x d \lambda
$$

and both of these intergals are absolutely convergent in view of $\left(H_{0}\right)$. We define a linear operater $J^{\alpha}$ such that:

$$
J^{\alpha} x=\frac{\sin \pi \alpha}{\pi} \int_{0}^{\infty} \lambda^{\alpha-1} R(\lambda, A)(-A) x d \lambda, \quad 0<\mathfrak{R e} \alpha<1 .
$$

For $0<\Re e ~ \alpha<2$, we define for each $x \in D\left(A^{2}\right)$

$$
\begin{aligned}
J^{\alpha} x= & \frac{1}{\Gamma(1-\alpha) \Gamma(\alpha)} \int_{0}^{\infty} \lambda^{\alpha-1}\left[R(\lambda, A)-\frac{\lambda}{1+\lambda^{2}}\right](-A) x d \lambda \\
& +\sin \pi \alpha / 2(-A) x .
\end{aligned}
$$

For $x \in D\left(A^{2}\right)$ definitions (2.1) and (2.2) coincide for overlapping ranges of $\alpha$. More generally, for $\alpha$ such that $n-1<\Re e \alpha<n$, we define, for $x \in D\left(A^{n}\right)$ :

$$
J^{\alpha} x=J^{\alpha-n+1}(-A)^{n-1} x .
$$

For $n-1<\Re e \alpha \leq n$, we define for $x \in D\left(A^{n+1}\right)$

$$
J^{\alpha} x=J^{\alpha-n+1}(-A)^{n-1} x .
$$

These definitions are also evidently consistent. In (2.1), the principal value of $\lambda^{\alpha}$ is taken so that $\lambda^{\alpha}$ is positive for $\alpha$ positive.

We shall now obtain some properties of these operators which will qualify them to be recognized as fractional powers. First, if $A$ does 
generate a semigroup, these coincide with the previous definitions in [1]. In particular, if we specialize $-A$ to be denote multiplication by the complex number $s$, non-negative, on the space of complex numbers, the definitions yield $s^{\alpha}$, principal determination.

Lemma 2.1. The operators $J^{a}$ can be extended to be closed linear.

Proof. The operators $J^{\alpha}$ being linear, it is enough to show that for any sequence $x_{n}, x_{n} \in D\left(J^{\alpha}\right)$, converging to zero, the sequence $J^{\alpha} x_{n}$, if convergent, has zero limit also. To be specific, let $0<\Re e \alpha<1$. Consider

$$
y_{n}=R(\lambda, A) J^{\alpha} x_{n},
$$

for fixed $\lambda$. Now $R(\lambda, A) x_{n} \in D(A)=D\left(J^{\alpha}\right)$ and it readily follow from (2.1) that

$$
R(\lambda, A) J^{\alpha} x_{n}=J^{\alpha} R(\lambda, A) x_{n} .
$$

Moreover, since $A R(\lambda, A)$ is bounded linear, so is $J^{\alpha} R(\lambda, A)$. Hence if limit $J^{a} x_{n}=y$, we have from (2.5) that $R(\lambda, A) y$ is zero, hence $y$ is zero also. The proof for other values of $\alpha$ is similar.

Lemma 2.2. For $x \in D\left(A^{n}\right), J^{\alpha} x$ is analytic in $\alpha$ for $0<\Re e ~ \alpha<n$.

Proof. This may be directly verified from the definitions. In particular, it may be noted that for $x \in D\left(A^{\infty}\right), J^{\alpha} x$ is analytic for $\Re(\alpha>0$.

For elements in certain domains larger than the ones in Lemma 2.2 , we retain continuity. Thus

Lemma 2.3. Let $x \in D(A)$ and $A x \in \overline{D(A)}$. Then for $0<$ Re $\alpha<1$ and $\alpha$ tending to 1 in a fixed sector about $1, J^{\alpha} x \rightarrow-A x$.

Proof. We note that since $A x \in \overline{D(A)}, \lambda R(\lambda, A) A x \rightarrow A x$ as $\lambda \rightarrow \infty$. Now

$$
J^{\alpha} x-(-A) x=\int_{0}^{\infty} \frac{\sin \pi \alpha}{\pi} \lambda^{\alpha-1}\left[R(\lambda, A)-\frac{1}{\lambda+1}\right](-A) x d \lambda,
$$

and the integral can be split into two parts, one from 0 to $L$ and the other from $L$ to infinity. For fixed $L$, the first part goes to zero since it is $0(|\sin \pi \alpha|)$. The second part in absolute value is

$$
\begin{gathered}
<L^{\sigma-1}\left|\frac{\sin \pi(1-\alpha)}{\pi(1-\sigma)}\right| \sup \|[\lambda R(\lambda, A)-I](-A) x\|+M \frac{L^{\sigma-2}}{|\sigma-2|}\|A x\|, \\
\sigma=\Re \mathrm{e} \alpha .
\end{gathered}
$$


and hence goes to zero also.

We do not in general have convergence to the identity at the origin, $\alpha=0$. For if for some $x, A x=0, J^{\alpha} x$ is zero also. However, we can state:

Lemma 2.4. For any $x \in D(A)$ such that $\lambda R(\lambda, A) x \rightarrow 0$ as $\lambda \rightarrow 0$, $J^{\alpha} x \rightarrow x$ as $\alpha \rightarrow 0+$ in a fixed sector about 0 .

Proof. We have

$$
\begin{aligned}
J^{\alpha} x-x & =-\frac{\sin \pi \alpha}{\pi} \int_{0}^{\infty}\left[R(\lambda, A) A x+\frac{x}{\lambda+1}\right] \lambda^{\alpha-1} d \lambda \\
& =-\frac{\sin \pi \alpha}{\pi} \int_{0}^{\infty} \lambda^{\alpha} \frac{R(\lambda, A)(x+A x)}{\lambda+1} d \lambda
\end{aligned}
$$

and the result follows from the second integral as a simple estimation shows.

Lemma 2.5. Let $x \in D\left(A^{2}\right)$. Then for $0<\Re e(\alpha+\beta)<1$,

$$
J^{\alpha+\beta} x=J^{\alpha} J^{\beta} x
$$

Proof. For $x \in D\left(A^{2}\right)$, it is clear from (2.1) that $J^{\beta} x \in D(A)=$ $D\left(J^{\alpha}\right)$. Moreover we have:

$$
J^{\alpha} J^{\beta} x=\frac{\sin \pi \alpha}{\pi} \frac{\sin \pi \beta}{\pi} \int_{0}^{\infty} \int_{0}^{\infty} \lambda^{\beta-1} \mu^{\alpha-1} R(\lambda, A) R(\mu, A) A^{2} x d \lambda d \mu,
$$

where the double integral is absolutely convergent, and can be rewritten as

$$
\frac{\sin \pi \alpha}{\pi} \frac{\sin \pi \beta}{\pi} \int_{0}^{1}\left(\sigma^{\beta-1}+\sigma^{\alpha-1}\right) d \sigma \int_{0}^{\infty} R(\lambda \sigma, A) R(\lambda, A) A^{2} x \lambda^{\alpha+\beta-1} d \lambda .
$$

Using the first resolvent equation, we have

$$
R(\lambda \sigma, A) R(\lambda, A) A^{2} x=\frac{\sigma R(\lambda \sigma, A)-R(\lambda, A)}{1-\sigma}(-A) x
$$

so that we have finally, after a change of variable:

$$
J^{\alpha} J^{\beta} x=\text { Const. } \int_{0}^{\infty} \lambda^{\alpha+\beta-1} R(\lambda, A)(-A) x d \lambda .
$$

where the constant

$$
\frac{\sin \pi \alpha}{\pi} \frac{\sin \pi \beta}{\pi} \int_{0}^{1} \frac{\left(\sigma^{\beta-1}+\sigma^{\alpha-1}-\sigma^{-\alpha}-\sigma^{-\beta}\right)}{(1-\sigma)} d \sigma
$$

evaluates to $\sin \pi(\alpha+B) / \pi$, thus verifying (2.6), 
The semigroup property is readily extended to all exponents for $x \in D\left(A^{\infty}\right)$. For exponents less than some finite positive number this domain can be enlarged. First, however, we define:

$$
(-A)^{\alpha}=\text { Smallest closed extension of } J^{\alpha} \text {. }
$$

We term this the principal value, even though we cannot, in general, claim that any other determination will differ from it by only a factor of exp $i \pi k$ for integral $k$. For an example see [1]. On the other hand, the principal determination still enjoys a uniqueness property similar to the one obtained by Hille [4] for linear bounded operators. We state this as a Lemma:

\section{LEMma 2.5. Let $x \in D\left(A^{\infty}\right)$. Then}

$$
\limsup _{|\eta| \rightarrow \infty} \frac{\log \left\|(-A)^{\sigma+i \eta} x\right\|}{|\eta|}<\pi, \quad 0<\sigma .
$$

Moreover, it is not possible to find a determination of $(-A)^{\alpha}$ analytic in $\alpha$ for $x \in D\left(A^{\infty}\right)$, interpolating integral powers, and preserving the extremal property, other than the one given in (2.7).

Proof. A direct calculation yields (2.8). The uniqueness part follows as in [4, p. 496], using a classical result of F. Carlson.

We note that all these fractional powers are uniquely determined by their values on $D\left(A^{n}\right)$ each for a large enough $n$. [Actually on $D\left(A^{\infty}\right)$, the latter domain being dense in $\overline{D(A)}$. See $\S 3$, Lemma 3.1.] The semigroup property (2.6) can be sharpened to read

$$
(-A)^{\alpha+\beta}=\left[(-A)^{\alpha}(-A)^{\beta}\right]_{c},
$$

the right side being the smallest closure of $(-A)^{\alpha}(-A)^{\beta}$. This follows essentially from the fact that $J^{\alpha}$ (and hence $(-A)^{\alpha}$ ) commutes with $R(\lambda, A)$, as in [1].

3. Spectral theory. We next examine the spectra of the operators $(-A)^{\alpha}$. For this purpose we denote the second commutant of the set $\{R(\lambda, A), \lambda>0\}$ by $\mathfrak{B}$. Then $\mathfrak{B}$ is a commutative Banach algebra with unit, and is strongly closed. Moreover, using the Gelfand theory, the linear multiplicative functionals over $\mathfrak{B}$ split into two classes $\mathfrak{M}_{0}$ and $\mathfrak{M}_{1}$. For any $m \in \mathfrak{M}_{0}$,

$$
m[R(\lambda, A)]=0
$$

while for any $m \in \mathfrak{M}_{1}$, there is an $s \in \sigma[A]$, such that

$$
m[R(\lambda, A)]=1 /(\lambda-s)
$$


for every $\lambda>0$.

For any function $\alpha$ of bounded variation on compact Borel sets of the half-line $[0, \infty)$ such that

$$
\int_{0}^{\infty}\|R(\lambda, A) x\| d|\alpha|<\infty
$$

it is clear that setting

$$
\theta(\alpha) x=\int_{0}^{\infty} R(\lambda, A) x d \alpha
$$

$\theta(\alpha) \in \mathfrak{B}$. We now collect some special functions we shall need in the sequel. Let $\mu$ be a complex number such that $\mu+|s|^{\alpha} \exp i \alpha \theta \neq 0$, for any $|s|$ and $-\pi \leq \theta \leq \pi$, and fixed $\alpha, 0<\Re e \quad \alpha<1$. We can always find such a number for $|\alpha|$ sufficiently small, and we shall assume this is the case. Let

$$
f(\lambda)=(1 / \pi)(1 / 2 i)\left[\left(\mu+\lambda^{\alpha} e^{-i \pi \alpha}\right)^{-1}-\left(\mu+\lambda^{\alpha} e^{i \pi \alpha}\right)^{-1}\right] .
$$

Next, for each $t>0$, let

$$
g(\lambda ; t)=(1 / \pi) \mathfrak{\Im m} \cdot\left[\exp \left(-t \lambda^{\gamma} \exp -i \pi \gamma\right)\right]
$$

for some fixed $\gamma, 0<\gamma<1 / 2$. Then

$$
\theta(f)=\int_{0}^{\infty} R(\lambda, A) f(\lambda) d r
$$

and

$$
O(g(\lambda ; t))=S(t)=\int_{0}^{\infty} R(\lambda, A) g(\lambda ; t) d \lambda
$$

both belong to $\mathfrak{B}$, the integrals existing in the Bochner sense in the uniform topology. Moreover, for $m \in \mathfrak{M}_{1}$, with corresponding $-s \in \sigma(A)$, we have

$$
m(\theta(f))=\int_{0}^{\infty} \frac{f(\lambda)}{\lambda+s} d \lambda=\left[\mu+s^{\alpha}\right]^{-1} .
$$

The integral on the right is of course the Stieltjes transform and exists for any $s$ not on the negative real axis. [For the properties of Stieltjes transforms explicitly or implicitly used here see [7].] On the other hand, we note that the spectrum of $A$ may be empty. Similarly,

$$
m(S(t))=\int_{0}^{\infty} \frac{g(\lambda ; t)}{\lambda+s} d \lambda=\exp -t s^{\gamma} .
$$

[Here and throughout, $s^{\alpha}=|s|^{\alpha} \exp i \alpha \theta,-\pi<\theta<\pi$ ].

Lemma 3.1. For every $x \in X, S(t) x \in D\left(A^{\infty}\right)$ for every $t>0$. For 
$x \in \overline{D(A)},\|S(t) x-x\| \rightarrow 0$ as $t \rightarrow 0+$

Proof. We note that for every positive integer $n$,

$$
\int_{0}^{\infty} \lambda^{n}|g(\lambda, t)|\|R(\lambda, A)\| d \lambda<\infty
$$

and

$$
\int_{0}^{\infty} \lambda^{n} g(\lambda ; t) d \lambda=0 \text {, including } n=0 \text {. }
$$

For any $x \in X$,

$$
A R(\lambda, A) x=\lambda R(\lambda, A) x-x .
$$

Hence

$$
\begin{aligned}
A S(t) x & =\int_{0}^{\infty} R(\lambda, A) g(\lambda ; t) d \lambda-x \int_{0}^{\infty} g(\lambda ; t) d \lambda \\
& =\int_{0}^{\infty} R(\lambda, A) \lambda g(\lambda ; t) x d \lambda
\end{aligned}
$$

using (3.5) and (3.6). In a similar manner we can extend this to any positive integer $n$.

$$
A^{n} S(t) x=\int_{0}^{\infty} \lambda^{n} R(\lambda, A) x g(\lambda ; t) d \lambda .
$$

This shows that $S(t) x \in D\left(A^{\infty}\right)$. Next let $x \in D(A)$. Now

$$
S(t) x-x=\int_{0}^{\infty} g(\lambda ; t)\left[R(\lambda, A)-\frac{1}{\lambda}\right] x d \lambda
$$

where we have used

$$
\int_{0}^{\infty} \lambda^{-1} g(\lambda ; t) d \lambda=1
$$

Since $x \in D(A)$, we can rewrite (3.8) as

$$
S(t) x-x=\int_{0}^{\infty} g(\lambda, t) \lambda^{-1} R(\lambda, A) A x d \lambda
$$

and the integral on the right is seen to go to zero with $t$. To see that the result is true for $x \in \overline{D(A)}$, we have only to note that (using $H_{0}$ ) $\|S(t)\| \leq M$.

Now, since $(-A)^{\alpha}$ is closed and $S(t) x \in D\left(A^{\infty}\right)$ for every $x,(-A)^{\alpha} S(t)$ is linear bounded and $\in \mathfrak{B}$. Actually, more is true. Thus:

LEMMA 3.2 .

$$
(-A)^{\alpha} S(t)=\int_{0}^{\infty} R(\lambda, \mathrm{A}) h(\lambda, t) d \lambda, \quad 0<\Re \mathrm{e} \alpha<1,
$$


where

$h(\lambda, t)=\frac{1}{2 \pi i}\left[e^{-i \pi \alpha} \lambda^{\alpha} \exp \left(-t \lambda^{\gamma} \exp -i \pi \gamma\right)-e^{i \pi \alpha} \lambda^{\alpha} \exp \left(-t \lambda^{\gamma} \exp +i \pi \gamma\right)\right]$.

Proof. We note that for $m \in \mathfrak{M}_{1}$,

$$
m\left[\int_{0}^{\infty} R(\lambda, A) h(\lambda, t) d \lambda\right]=\int_{0}^{\infty} \frac{h(\lambda, t)}{\lambda+s} d \lambda
$$

and the Stieltjes transform on the right evaluates to

$$
s^{\alpha} \exp \left(-t s^{\alpha}\right) \text {. }
$$

While this is the same as $m\left[(-A)^{\alpha} S(t)\right]$, this does not necessarily constitute a proof of (3.9) since the radical of $\mathfrak{B}$ may be non-empty. However, a direct proof is possible. Thus we have

$$
(-A)^{\alpha} S(t)=\int_{0}^{\infty} \int_{0}^{\infty} \frac{\sin \pi \alpha}{\pi} R(\lambda)(-A) R(\mu) \lambda^{\alpha-1} g(\mu, t) d \lambda d \mu
$$

and changing variable of integration and using

$$
R(\lambda \sigma, A)(-A) R(\lambda)=\frac{\sigma R(\lambda \sigma, A)-R(\lambda, A)}{1-\sigma}
$$

this can be written

$$
\int_{0: 1}^{\infty} R(\lambda, A) h(\lambda, t) d \lambda
$$

where

$$
h(\lambda, t)=g(\lambda, t) \int_{0}^{1} \frac{\sigma^{-\alpha}-\sigma^{\alpha-1}}{(1-\sigma)} d \sigma+\int_{0}^{1} \sigma^{-1} \frac{g(\lambda / \sigma)-g(\lambda \sigma)}{(1-\sigma)} d \sigma
$$

which is readily verified to be the function required in (3.9).

\section{LEMMA 3.3.}

$$
\theta(f)(-A)^{\alpha} S(t)=\int_{0}^{\infty} R(\lambda, A) q(\lambda, t) d \lambda
$$

where

$$
q(\lambda, t)=\frac{1}{2 \pi i}\left[\frac{\lambda^{\alpha} e^{-i \pi \alpha} \exp \left(-t \lambda^{\gamma} \exp -i \pi \gamma\right)}{\mu+\lambda^{\alpha} \exp -i \pi \alpha}-\frac{\lambda^{\alpha} e^{i \pi \alpha} \exp \left(-t \lambda^{\gamma} \exp i \pi \gamma\right)}{\mu+\lambda^{\alpha} \exp i \pi \alpha}\right] .
$$

Proof. The Stieltjes tranform of $q(\lambda, t)$ is readily verified to be

$$
\int_{0}^{\infty} \frac{q(\lambda, t) d t}{\lambda+s}=\frac{s^{\alpha} \exp -t s^{\gamma}}{\mu+s^{\alpha}}
$$


As in Lemma 3.2, this is not quite enough to prove (3.10). On the other hand, a direct proof may be given by double integration using the resolvent equation, and noting that

$q(\lambda, t)=\int_{0}^{1}\left(h(\lambda, t)\left(f(\lambda \sigma)-\sigma^{-1} f(\lambda / \sigma)\right)+f(\lambda)\left(h(\lambda \sigma, t)-\sigma^{-1} h(\lambda / \sigma, t)\right)\right) \frac{d \sigma}{\sigma-1}$.

LEMMA 3.4.

$$
\theta(f) S(t)=\int_{0}^{\infty} R(\lambda, A) r(\lambda, t) d \lambda
$$

where

$$
r(\lambda, t)=\frac{1}{2 \pi i}\left[\frac{\exp \left(-t \lambda^{\gamma} \exp -i \pi \gamma\right)}{\mu+\lambda^{\alpha} \exp -i \pi \alpha}-\frac{\exp \left(-t \lambda^{\gamma} \exp i \pi \gamma\right)}{\mu+\lambda^{\alpha} \exp i \pi \alpha}\right] .
$$

Proof. The Stieltjes transform of $r(\lambda, t)$ is

$$
\int_{0}^{\infty} \frac{r(\lambda, t)}{\lambda+s} d \lambda=\frac{\exp -t s^{\gamma}}{\mu+s^{\alpha}}
$$

As in Lemma 3.3, we can establish (3.11) by double integration; using the resolvent equation.

Lemma 3.5. Let $x \in \overline{D(A)}$. Then with $\mu$ as in Lemma 3.1,

$$
\left[\mu+(-A)^{\alpha}\right] \theta(f) x=x .
$$

Proof. From the previous Lemmas it is immediate that

$$
\mu \theta(f) S(t)+(-A)^{\alpha} \theta(f) S(t)=S(t)
$$

for every $t>0$. Let $x \in D(A)$. Since

$$
\left[\mu+(-A)^{\alpha}\right] \theta(f) S(t) x=S(t) x
$$

and by Lemma 3.2, $S(t) x \rightarrow x$ as $t \rightarrow 0$, (3.12) follows by letting $t \rightarrow 0$, and noting that $(-A)^{\alpha}$ is closed.

We are now ready to prove the spectral mapping theorem.

Theorem 3.1. Let $D(A)$ be dense in $X$. Then

$$
\sigma\left[(-A)^{\alpha}\right]=[\sigma(-A)]^{\alpha}, \quad \text { Re } \alpha>0 .
$$

Proof. First let $|\alpha|$ be so small that we can find a $\mu$ as in Lemma 3.1. By Lemma 3.5, for $X \in D(A)$, (3.12) holds, and $D(A)$ being dense in $X$, continues to hold for any $x$. Since for $x \in D\left((-A)^{\alpha}\right), \theta(f)(-A)^{\alpha} x=$ $(-A)^{\alpha} \theta(f) x$, we see that $\theta(f)$ is a resolvent of $-(-A)^{\alpha}, \theta(f)=R\left(u,-(-A)^{\alpha}\right)$. 
This is enough to prove that for these $\alpha$, (3.13) holds. For, let $\delta$ be a number different from $+\mu$ such that $-\delta \neq[\sigma(-A)]^{\alpha}$. Then consider $\left[I+(\delta-\mu) R\left(\mu,-(-A)^{\alpha}\right)\right]$. This belongs to $\mathfrak{B}$, and

$$
\begin{aligned}
m\left[I+(\delta-\mu) R\left(\mu,-(-A)^{\alpha}\right)\right] & =1 & & m \in \mathfrak{M}_{0} \\
& =\frac{\delta+s^{\alpha}}{\mu+s^{\alpha}} & & m \in \mathfrak{M}_{1} .
\end{aligned}
$$

Hence this element has an inverse in $\mathfrak{B}$, and this is easily seen to be $R\left(\delta,-(-A)^{\alpha}\right)$. For other values of $\alpha$, we note that for $\alpha$ such that $\rho\left[(-A)^{\alpha}\right]$ is not empty, we have a sharper version of (2.9):

$$
(-A)^{n \alpha}=\left[(-A)^{\alpha}\right]^{n} \text { for every integer } n \text {. }
$$

Again, by the general spectral mapping theorem for closed operators with a non-empty resolvent set [4], we see that (3.13) holds for $n \alpha$. Finally, we note that for $\alpha=a+i b$, and $a^{2}+b^{2}<a$, it is always possible to find a $\mu$ such $\mu+s^{\alpha} \neq 0$ for all $s$ not on the negative real axis.

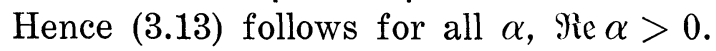

4. Some stability properties. We shall call a property of $A$ stable, if the same property holds for $-(-A)^{\alpha}$ at least for $0<\alpha<1$. We now state some stable properties of $A$.

4.1. Let $A$ be linear bounded. Then $-(-A)^{\alpha}$ is also bounded for every $\alpha$, গe $\alpha>0$.

4.2. Let $A^{*}$ be the adjoint of $A$. In view of hypothesis $H_{0}$ we can, following Phillips [4], define $A^{\odot}$ using his definition (Definition 14.3.1, p. 424). For $|\alpha|$ sufficiently small, we note that $-(-A)^{\alpha}$ also satisfies hypothesis $H_{0}$, so that we can also define $\left[(-A)^{\alpha}\right]^{\circ}$. We then have that

$$
\left[(-A)^{\alpha}\right]^{\odot}=\left[(-A)^{\bullet}\right]^{\alpha} \text {. }
$$

4.3. Let $A$ be the infinitesimal generator of a positive contraction semigroup. Then so is $-(-A)^{\alpha}$, for $0<\alpha<1$.

4.4. Let $X$ be a Hilbert space. If $A$ is dissipative, so is $-(-A)^{\alpha}$ for $0<\alpha<1$.

4.5. Let $A$ be compact. Then so is $(-A)^{\alpha}$ for every $\alpha$.

4.6. If for some $x \in X$, and $s$ not on the negative real axis $A x=$ $-s x$, then $(-A)^{\alpha} x=s^{\alpha} x$ also.

5. Generation of semigroups. We now come to what is perhaps 
the most important single property of these fractional powers (at least as far as applications to differential equations are concerned) viz., generation of semigroups. We shall show that for $0<\alpha \leq 1 / 2,-(-)^{\alpha}$ generate strongly continuous semigroups, uniformly continuous away from the origin. We shall also obtain a representation for these semigroups in terms of $R(\lambda, A), \lambda>0$.

Theorem 5.1. Let $D(A)$ be dense, and let $A$ satisfy $H_{0}$. Then for $0<\alpha \leq 1 / 2,-(-A)^{\sigma}$ as defined by 2.7, generates a semigroup $S_{a}(t)$, which is strongly continuous for $t \geq 0$, uniformly continuous for $t>0$.

Proof. First let $0<\alpha<1 / 2$. Let

$$
S_{\alpha}(t)=\int_{0}^{\infty} R(\lambda, A) g(\lambda, t ; \alpha) d \lambda
$$

where

$$
g(\lambda, t ; \alpha)=(1 / \pi) \sin \left(t \lambda^{\alpha} \sin \pi \alpha\right) \exp \left(-t \lambda^{\alpha} \cos \pi \alpha\right) .
$$

Then (5.1) is a Bochner integral. The Stieltjes transform of $g(\lambda, t ; \alpha)$ is, as we have noted before in $\S 3$,

$$
\int_{0}^{\infty} \frac{1}{\lambda+s} g(\lambda, t ; \alpha) d \lambda=\exp -t s^{\alpha} .
$$

However, this alone does not necessarily suffice to verify the semigroup property of $S_{a}(t)$. A direct proof can be given, however, following the lines of Lemma 3.3. We shall next show that the infinitesimal generator of $S_{a}(t)$ is $-(-A)^{\alpha}$, by showing that the resolvent of the latter for $\mu>0$, is the Laplace transform of $S_{\alpha}(t)$. The Laplace transform can, further, be taken in the uniform topology, since $S_{a}(t)$ is readily seen to be uniformly continuous for $t>0$, by direct computation from (5.1). Now,

$$
\begin{aligned}
\int_{0}^{\infty} e^{-\mu t} S_{\alpha}(t) d t & =\int_{0}^{\infty} R(\lambda, A) \int_{0}^{\infty} e-{ }^{\mu t} g(\lambda, t ;) d t d \lambda \\
& =\int_{0}^{\infty} R(\lambda, A)\left[\frac{1}{\mu+\lambda^{\alpha} e^{-i \pi \alpha}}\right] d \lambda
\end{aligned}
$$

which as we have seen in Lemma 3.5, is the resolvent of $-(-A)^{\alpha}$ for $\mu>0$. The strong continuity of $S_{\alpha}(t)$ has already been proved in Lemma 3.1 .

Next let $\alpha=1 / 2$. Let

$$
S_{1 / 2}(t)=\int_{0}^{+\infty} R(\lambda, A) \sin \sqrt{\lambda} t d \lambda,
$$

where the integral is to be taken at infinity in the Cauchy sense. The 
convergence in the Cauchy sense can be seen as follows. By an integration by parts, we have, for each $L$,

$$
\frac{1}{\pi} \int_{0}^{L} R(\lambda, A) \sin \sqrt{\lambda} t d \lambda=R(L, A) f(L, t)+\int_{0}^{L} R(\lambda, A)^{2} f(\lambda, t) d \lambda,
$$

where

$$
f(\lambda, t)=\frac{1}{\pi} \int_{0}^{\lambda} \sin \sqrt{\sigma} t d \sigma=\frac{1}{\pi}\left[\frac{2 \sin \sqrt{\lambda} t}{t^{2}}-\frac{2 \sqrt{\lambda} \cos \sqrt{\lambda} t}{t}\right] .
$$

Now the first term goes to zero as $L \rightarrow \infty$, and the second term is a convergent Bochner integral at infinity. Hence

$$
S_{1 / 2}(t)=\frac{1}{\pi} \int_{0}^{\infty} R(\lambda, A) \sin \sqrt{\lambda} t d \lambda=\int_{0}^{\infty} R(\lambda, A)^{2} f(\lambda, t) d \lambda .
$$

Next, $S_{1 / 2}(t)$ is readily seen to be uniformly continuous for $t>0$. A simple computation using (5.3) also shows that $\left\|S_{1 / 2}(t)\right\| \leq$ Const. The semigroup property can be verified directly as before. Again, the Laplace transform of $S_{1 / 2}(t)$ is seen to be the resolvent of $-(-A)^{1 / 2}$. The strong continuity at the origin may be seen from:

$$
\left\|S_{1 / 2}(t) x-x\right\|=\left\|\frac{1}{\pi} \int_{0}^{\infty}\left[R(\lambda, A)-\frac{1}{\lambda}\right] x \sin \sqrt{\lambda} t d \lambda\right\| .
$$

For fixed $L$ and $t$ sufficiently small, the first term is $O(t)$. The second term is

$$
\leq\|A x\| M \int_{L}^{\infty} \frac{\mid \sin \sqrt{\lambda} t}{\lambda^{2}} d \lambda
$$

and hence goes to zero also. Since $\left\|S_{1 / 2}(t)\right\|$ is bounded, strong continuity follows. This completes the proof of the theorem.

For values of $\alpha>1 / 2,-(-A)^{\alpha}$ does not necessarily generate a semigroup of any type, as the following simple counter-example shows. Let $X=l_{2}(-\infty, \infty)$, and let $A$ correspond to multiplying the $n$th coordinate by $(1+i) n$. For $\alpha>1 / 2$, no right half plane is free of spectra of $-(-A)^{\alpha}$, (as follows readily from Theorem 3.1 ) so that they cannot be generators of any semigroup.

We note in passing that (5.1) leads to a simple rigorous proof of Feller's expansion for the stable densities [3] for $0<\alpha \leq 1 / 2$. For, denoting the stable density by $F(\xi, t ; \alpha)$, we have

$$
F(\xi, t ; \alpha)=\frac{1}{\pi} \int_{0}^{\infty} e^{-\xi \lambda} \Im \mathrm{m}\left[\exp \left(-t \lambda^{\alpha} \exp -i \pi \alpha\right)\right] d \lambda
$$

and expanding the second factor and interchanging integration and summation, which is obviously permissible, we have 


$$
F(\xi, t ; \alpha)=-\frac{1}{\pi} \sum_{0}^{\infty} \frac{(-t)^{n}}{n !} \xi^{-n \alpha-1} \sin \pi \alpha n I^{\prime}(1+n \alpha)
$$

which is Feller's expansion.

6. Application to abstract Cauchy problems. We shall next consider an application of the foregoing theory to a class of Cauchy problems. Indeed, this was the application which largely motivated the theory. This class may be considered a generalization of the abstract Cauchy problem of the type

$$
u(t)=A u(t)
$$

and is related to (though different from) the class treated by Hille $[4,5]$. Thus we shall examine abstract Cauchy problems of the type

$$
\frac{d^{n} u(t)}{d t^{n}}+(-1)^{n} A u(t)=0, \quad n \geq 2 .
$$

More precisely, we shall phrase the problem as follows:

Given a complex Banach space $X$, and a closed linear operator $A$ with domain dense in $X$ and range in $X$, find a function $u(t)$ such that

(i) $u(t)$ is $n$ times continuously differentiable in $[0, \infty)$

(ii) $u(t) \in D(A)$ for $t \geq 0$

(iii) $u(t)$ satisfies (6.1) for $t>0$, and the initial conditions

$$
\lim _{t \rightarrow 0}\left\|u^{k}(t)-u_{k}\right\|=0 \text { for prescribed } u_{k}, k=0,1, \cdots, r, \quad r \leq n .
$$

This is the reduced problem ('problème reduit') in the terminology of Hille [5, p. 42], $n-r$ being the defect ('default'). In addition to the existence of solutions [with some defect], we are of course interested in the uniqueness of the solutions. Now, if the operator $A$ satisfies $H_{0}$, we are [by Theorem 5.1] assured of solutions for some suitable defect, but the question of uniqueness remains. On the other hand, if we do have unique solutions for some $A$, we would certainly like to know whether this implies that $A$ satisfy $H_{0}$, since this would then characterize the solutions completely. In what follows we are concerned exclusively with the case $n=2$. Our main result may be stated as follows:

Theorem 6.1. Let $n=2$. Suppose $A$ satisfies $H_{0}$. Then for each $u_{0} \in D(A)$, the reduced problem with defect one has a solution such that

$$
\sup _{t \geq 0}\|u(t)\|<\infty \text {. }
$$

Moreover, there is only one such solution, and it is given by

$$
u(t)=S_{1 / 2}(t) u_{0}
$$


where the semigroup $S_{1 / 2}(t)$ is uniformly continuous for $t>0$, and has the representation (5.3). Further, for each $t>0$, range of $S_{1 / 2}(t) \subset D(A)$ and $S_{1 / 2}(t)$ is analytic of class $H(\phi,-\phi), \phi>0$. Conversely, suppose for each $u_{0} \in D(A)$, the reduced problem has a unique solution satisfying (6.2) for some $A$. Then setting $u(t)=S(t) u_{0}$, yields $S(t)$ as a strongly continuous semigroup. Suppose range of $S(t) \subset D(A)$, and $S(t)$ $i$ analytic of class $H(\phi,-\phi)$ for some $\phi>0$. Then $A$ satisfies $H_{0}$, and $S(t)=S_{1 / 2}(t)$ as given by (5.3).

We need some Lemmas.

Lemma 6.1. Let $A$ satisfy $H_{0}$. Then $R(\lambda, A)$ exists in the sector $-\phi<\arg \lambda<\phi$, where $(\operatorname{Tan} \phi) M=1$.

Proof. Let $\varepsilon>0$, and $\gamma_{\varepsilon} M=1-\varepsilon$. For any $t,|t|<\gamma_{\varepsilon}$, and $\sigma>0$ consider $I+[(\sigma+i t \sigma)-\sigma] R(\sigma, A)$. This element has an inverse given by the series

$$
\sum_{0}^{\infty}[-i t \sigma R(\sigma, A)]^{n}
$$

and is clearly convergent, being majorized by the geometric series

$$
\sum_{0}^{\infty}|t|^{n} M^{n}<\frac{1}{1-\gamma_{\varepsilon} M} .
$$

Moreover this also shows that the inverse is bounded in norm by $\left(1-\gamma_{\varepsilon} M\right)^{-1}$. Now, by the first resolvent equation, it follows that

$$
R(\sigma+i t \sigma, A)=[I-[(\sigma+i t \sigma)-\sigma] R(\sigma, A)]^{-1} R(\sigma, A)
$$

and is in norm

$$
\|R(\sigma+i t \sigma, A)\| \leq \frac{M}{\left(1-\gamma_{\varepsilon} M\right) \sigma} .
$$

The assertion of the lemma follows readily from this.

Lemma 6.2. Suppose $A$ satisfies hypothesis $H_{0}$. Then for each $\alpha$, $0 \leq \alpha \leq 1 / 2, \lambda \in \rho\left[-(-A)^{\alpha}\right]$ for $-\psi-\pi / 2<\arg \lambda<\pi / 2+\psi$ for some $\psi>0$.

Proof. The proof is immediate from the spectral mapping theorem, Theorem 3.1, and Lemma 6.1 above. We can take $\psi=\pi / 2-\alpha(\pi-\phi)$.

Lemma 6.3. Let $A$ satisfy $H_{0}$. Then for $0<\alpha \leq 1 / 2$, the semigroup $S_{a}(t)$, defined by 5.1 and (5.3) is analytic, of class $H\left(\phi_{1}, \phi_{2}\right)$ (Cf [4], p. 325, Definition 10.6.1), with $\phi_{1}=-\alpha \varphi, \phi_{2}=\alpha \varphi, \varphi$ being defined in Lemma 6.1. 
Proof. Let $\mu$ be such that $\Re e \mu>0$. Then for each $\alpha, 0<\alpha \leq$ $1 / 2$, we know from Lemma 3.5 that $\mu \in \rho\left[-(-A)^{\alpha}\right]$ and

$$
R\left(\mu,-(-A)^{\alpha}\right)=\frac{1}{\pi} \int_{0}^{\infty} R(\lambda, A)\left[\frac{1}{\mu+\lambda^{\alpha} e^{-i \pi \alpha}}-\frac{1}{\mu+\lambda^{\alpha} e^{i \pi \alpha}}\right] d \lambda .
$$

From this it readily follows that

$$
|| R\left(\mu,-(-A)^{\alpha}\right) \mid \leq \frac{M}{\Re e \mu}
$$

where the constant $M$ is the same as in $H_{0}$. Next let $\varepsilon<0$ be given. Then from Lemma 6.1 it follows that for $-\phi+\varepsilon \leq \arg \lambda \leq-\varphi-\varepsilon$, there is a constant $M_{\varepsilon}$ such that

$$
\|\lambda R(\lambda, A)\| \leq M_{\varepsilon} \text {. }
$$

Let $-\varphi+\varepsilon \leq \psi \leq+\varphi-\varepsilon$. Let $\lambda$ be $>0$. Then

$$
R\left(\lambda, e^{-i \psi} A\right)=e^{i \psi} R\left(\lambda e^{i \psi r}, A\right) .
$$

Further it follows from (6.6) that

$$
\left\|\lambda R\left(\lambda, e^{-i \psi} A\right)\right\| \leq M_{\varepsilon},
$$

so that $(A \exp -i \psi)$ satisfies $H_{0}$. Then we can define $\left(-e^{-i \psi} A\right)^{\alpha}$ using (2.7), and a simple contour integration shows that for $0<\alpha<1$,

$$
\left(-e^{-i \psi} A\right)^{\alpha}=e^{-i \psi \alpha}(-A)^{\alpha} .
$$

Moreover, applying (6.5) we know that for $\Re e{ }>0$,

$$
\left\|R\left(\mu,-e^{-i \psi \alpha}(-A)^{\alpha}\right)\right\| \leq \frac{M_{\varepsilon}}{\Re e \mu} .
$$

But

$$
\left\|R\left(\mu,-e^{-i \psi \alpha \alpha}(-A)^{\alpha}\right)\right\|=\left\|R\left(\mu e^{i \psi \alpha \alpha},-(-A)^{\alpha}\right)\right\| .
$$

Hence we obtain that for $\lambda$ such that $\mathfrak{R e}\left(\lambda e^{-i \psi \alpha \alpha}\right)>0$,

$$
\left\|R\left(\lambda,-(-A)^{\alpha}\right)\right\| \leq \frac{M_{\varepsilon}}{\mathfrak{R e}\left(\lambda e^{-i \gamma \alpha \alpha}\right)} .
$$

But this implies that the conditions for $S_{\alpha}(t)$ to be of class $H\left(\phi, \phi_{2}\right)$ as given by Hille ([4] p. 383, Theorem 12.8.1) are satisfied, thus proving lemma.

Proof of Theorem. We begin with the first part. Thus let $A$ satisfy $H_{0}$. Setting $u(t)=S_{1 / 2}(t) u_{0}$, we get one solution satisfying (6.2). We shall now show that this solution is unique. Let $v(t)$ be a possibly 
different solution. By assumption $v^{\prime}(t)$ is continuous at $t=0$. Let $v_{1}$ be $v^{\prime}(0)$. Let $w_{0}=S(1) u_{1}$, and $w_{1}=S(1) v_{1}$, where $S(t)$ is defined as in Lemma 3.1. Let $w(t)=S(1) v(t)$. Then $w(t), w^{\prime}(t) \in D\left(A^{\infty}\right)$ and

$$
w^{\prime \prime}(t)+A w(t)=0 \text {. }
$$

Let

$$
L(\lambda, w)=\int_{0}^{\infty} e^{-\lambda t} w(t) d t
$$

Then since

$$
w^{\prime}(t)=w_{1}+A S(1) \int_{0}^{t} u(\sigma) d \sigma
$$

we get that $w^{\prime}(t) \exp -\lambda t$ goes to zero at infinity, and hence by Laplace transforming (6.7) we have:

$$
\left[\lambda^{2}+A\right] L(\lambda, w)=\lambda w_{0}+w_{1} .
$$

Since $B^{2}=-A$, where we have written $B$ for $-(-A)^{1 / 2}$, this can be rewritten

$$
[\lambda I-B] L(\lambda, w)=R\left(\lambda w_{0}+w_{1}\right)
$$

Since

$$
R(\lambda, B)=\int_{0}^{\infty} e^{-\lambda t} S_{1 / 2}(t) d t
$$

this yields

$$
w^{\prime}(t)+B w(t)=S_{1 / 2}(t) B w_{0}+S_{1 / 2}(t) w_{1} .
$$

Hence

$$
\frac{d}{d t}\left[S _ { 1 / 2 } \left(t(w(t)]=S_{1 / 2}(2 t) w_{1}+S_{1 / 2}(2 s) B w_{0}\right.\right.
$$

so that

$$
\begin{aligned}
S(t) w(t) & =w_{0}+\int_{0}^{t} S_{1 / 2}(2 t) w_{1} d t+\int_{0}^{t} S_{1 / 2}(2 t) B w_{0} d t \\
& =w_{0}+\frac{1}{2} \int_{0}^{2 t} S_{1 / 2}(\sigma) w_{1} d \sigma+\frac{1}{2}\left(S_{1 / 2}(2 t) w_{0}-w_{0}\right) \\
& =S_{1 / 2}(2 t) w_{0}-\frac{1}{2} \int_{0}^{2 t} S_{1 / 2}(\sigma)\left(w_{1}-B w_{0}\right) d \sigma .
\end{aligned}
$$

Hence

$$
S_{1 / 2}(t) B w(t)=S_{1 / 2}(2 t) w_{0}+\frac{1}{2}\left[S_{1 / 2}(2 t)-I\right]\left(w_{1}-B w_{0}\right)
$$

Now because of analyticity in a sector, zero does not belong to the point 
spectrum of $S_{1 / 2}(t)$ for any $t>0$. Writing $S_{1 / 2}(-t)$ for the inverse, and using (6.2), we have that

$$
\operatorname{Sup}\left\|S_{1 / 2}(-t)\left(w_{1}-B w_{0}\right)\right\|<\infty .
$$

We shall now show that for any element $z$ such that $z \in \bigcap_{t} D\left(S_{1 / 2}(-t)\right)$ and $\operatorname{Sup}\left\|S_{1 / 2}(-t) z\right\|<\infty, B z=0$. For this let

$$
F(\lambda)=\int_{0}^{\infty} e^{\lambda t} S_{1 / 2}(-t) z d t, \quad \Re \mathrm{ie} \lambda<0 .
$$

Then it is readily verified that

$$
[\lambda I-B](-F(\lambda))=z .
$$

Now by Lemma 6.2 , we know that $R(\lambda, B)$ exists for $-\psi-\pi / 2<$ $\arg \lambda<\psi+\pi / 2$ and hence there is a common domain where $-F(\lambda)=$ $R(\lambda, B) z$. Hence for $\Re(\lambda<0,-F(\lambda)$ is the analytic continuation of $R(\lambda, B)$. Moreover, using the results of Lemma 6.3 , it follows that $\|\lambda F(\lambda)\| \leq$ const., in a sector and $\|\lambda R(\lambda, B) z\| \leq$ const. in an intersecting sector, their union being the entire plane. Hence it follows that $\lambda R(\lambda, B) z=z$, since $\lambda R(\lambda, B) z \rightarrow z$ for $\lambda>0$. Hence $B z=0$, as required. Hence $S_{1(2)}(t) z=z$, so that

$$
S(t) w(t)=S(2 t) w_{0}-t\left(w_{1}-B w_{0}\right) .
$$

Hence using (6.2), $w_{1}=B w_{0}$. Since $u_{0} \in D(A)$, using $S(1 / n)$ in place of $S(1)$ and taking limits, we readily obtain that

$$
v^{\prime}(0)=B u_{0}
$$

and hence that $v(t)=S_{1 / 2}(t) u_{0}$. That range at $S_{1 / 2}(t) \subset D(A)$ follows from the representation (5.3).

We now proceed to the second part of the theorem. That $S(t)$ is a semigroup, strongly continuous at the origin with $\|S(t)\| \leq$ const., follows by arguments similar to the one used in Lemma 23.9 .4 p. 627 of [4]. Let $B$ be the infinitesimal generator of $S(t)$. Then for $x \in D(A)$, it is clear $B^{2} x=-A x$. For $x \in D\left(B^{2}\right)$ on the other hand, we note that since for $t>0, S(t) x \in D(A), B^{2} S(t) x=-A S(t) x$, so that letting $t \rightarrow 0$, it follows that $B^{2} x=-A x$ also, since $A$ is closed. Next we note that

$$
\lambda^{2}-A=(i \lambda-B)(i \lambda+B)
$$

so that for $\lambda>0, \lambda \in \rho(A)$ since $S(t)$ is analytic, and

$$
R(\lambda, A)=R(i \sqrt{\lambda}, B) R(-i \sqrt{\lambda}, B) .
$$

Again since $S(t)$ is analytic, of class $H(\phi,-\phi)$, it readily follows that $\|\lambda R(i \lambda, B)\| \leq$ Const., for $\lambda$ real, from which we obtain that $\|\lambda R(\lambda, A)\| \leq$ Const., for $\lambda>0$. Or, $A$ satisfies hypothesis $H_{0}$. That $S(t)=S_{1 / 2}(t)$ is 
immediate from the first part of the theorem.

Additional properties of the solutions can of course be deduced from the representation (5.3). For instance, we note a rate of growth property: viz., for each $x \in X,\left\|S_{1 / 2}(t) x\right\| \rightarrow 0$ as $t \rightarrow \infty$, if $\lambda R(\lambda) x \rightarrow 0$ as $\lambda \rightarrow 0+$.

7. Some extensions. In this section we shall indicate some possible extensions of the foregoing theory.

The basic hypothesis $H_{0}$ concerning the operator $A$ can be weakened. Thus suppose $A$ satisfies $H_{1}$ :

For each $\lambda>0, \lambda \in \rho(A)$ and $\left(H_{1}\right)$

(i) $\|R(\lambda, A)\|=0(1 / \lambda)$ as $\lambda \rightarrow \infty$

(ii) $\int_{0}^{1} \lambda^{\sigma}\|R(\lambda, A)\| d \lambda<\infty$, for some $\sigma, 0<\sigma<1$.

Then it is possible to define $(-A)^{\alpha}$ for $\Re(\alpha \geq \sigma$, still using definition (2.7). The hypothesis $H_{1}$ is satisfied for instance if $A$ generates a semigroup $T(\xi)$ such that it is strongly continuous for $\xi \geq 0$, and

$$
\int_{1}^{\infty}\|T(\xi)\| \xi^{-\sigma-1} d \xi<\infty \text {. }
$$

The latter condition was used in [1], whereas hypothesis $H_{1}$ is similar to the one stated by Hille [4] (although of course the Hille condition is stronger since he considered only bounded operators). We shall show that for infinitesimal generators, $H_{1}$ and (7.1) are equivalent.

Lemma 7.1. Suppose $A$ is the infinitesimal generator of a strongly continuous semigroup $T(\xi)$. Then if (7.1) holds, $A$ satisfies $H_{1}$. Conversely, if $A$ satisfies $H_{1}, T(\xi)$ satisfies (7.1).

Proof. Suppose (7.1) holds. Then clearly

$$
\lim _{\xi \rightarrow \infty} \frac{\log \|T(\xi)\|}{\xi} \leq 0
$$

so that $R(\lambda, A)$ exists for $\lambda>0$ and is of order $1 / \lambda$ for $\lambda \rightarrow \infty$. Next

$$
\begin{aligned}
\int_{0}^{1} \lambda^{\sigma}\|R(\lambda, A)\| d \lambda & \leq \int_{0}^{1} \lambda^{\sigma} \int_{0}^{\infty} e^{-\lambda \xi}\|T(\xi)\| d \xi d \lambda \\
& \leq \text { const }+\int_{1}^{\infty}\|T(\xi)\| d \xi \int_{0}^{1} e^{-\lambda \xi} \lambda^{\sigma} d \lambda \\
& \leq \text { const } \int_{1}^{\infty}\|T(\xi)\| \xi^{-\sigma-1} d \xi<\infty .
\end{aligned}
$$

To prove the converse we shall use some results from [1]. Let $S(\omega)$ be the $B$-algebra associated with $T(\xi)$ as in [1] $L(\omega)$ being the subspace of functions (Borel measurable) such that 


$$
\int_{0}^{\infty}\|T(\xi)\||f(\xi)| d \xi<\infty
$$

Let $A_{w}$ be the infinitesimal generator of the translation semigroup. Defining $\left(-A_{w}\right)^{\alpha}$ using (2.7) it follows for $f \in D\left(A_{w}\right)$, setting

$$
\left(-A_{w}\right)^{\alpha} f=g
$$

that

$$
(-\lambda)^{\alpha} \phi(\lambda, f)=\phi(\lambda, g), \quad \text { Re } \lambda \leq 0,
$$

and hence that for any $\mu>0$,

$$
\frac{(-\lambda)^{\alpha}}{\mu-\lambda}
$$

is a multiplier defined over all of $L(\omega)$. By the factor theorem (cf [1]), it follows that there is a corresponding function in $S(\omega)$ (actually in $L(\omega)$ ) and further an evaluation of this function shows that (7.1) is satisfied.

While for $\Re$ e $\alpha \geq \sigma$, we can define $(-A)^{\alpha}$, it is not possible to define, in general, $(-A)^{\alpha}$ for $\Re(\lambda<\sigma$, at least not as a closed operator whose domain includes the domain of $A$. This may be seen as in the converse part of Lemma 7.1 , using $\|T(\xi)\|=(1+\xi)^{\sigma}$.

For $A$ satisfying $H_{1}$ with $\sigma \leq 1 / 2,-(-A)^{\alpha}$ continues to generate strongly continuous semigroups for $\alpha \leq 1 / 2$, satisfying

$$
\lim _{t \rightarrow \infty} \frac{\log \left\|S_{\alpha}(t)\right\|}{t} \leq 0 \text {. }
$$

We do not know at present whether the semigroups are necessarily analytic of class $H\left(\phi_{1}, \phi_{2}\right)$.

\section{REFERENCES}

1. A. V. Balakrishnan, An operational calculus for infinitesimal generators of semigroups, Trans. A.M.S., 1959.

2. S. Bochner, Diffusion equation and stochastic processes, Proc. Nat. Acad. Sciences, U.S.A., 35 (1949), 368-370.

3. W. Feller, On a generalization of Marcel Riesz' potentials and the semigroups generated by them, Communications du séminaire mathématique de l'université de Lund, tome supplimentaire (1952), dedie' a Marcel Riesz.

4. E. Hille and R. S. Phillips, Functional analysis and semigroups, Amer. Math. Soc. Colloq. Publ., Vol. 31.

5. E. Hille, Une generalization du problème de Cauchy, Annales de L'Institut Fourier, Tome 4, 1952.

6. R. S. Phillips, On the generation of semigroups of linear operators, Pacific J. Math., (1952), 333-369.

7. D. V. Widder, The Laplace transform, Princeton University Press, 1946. 



\section{PACIFIC JOURNAL OF MATHEMATICS}

\section{EDITORS}

David Gilbarg

Stanford University

Stanford, California

F. H. Brownell

University of Washington

Seattle 5, Washington
A. L. Whiteman

University of Southern Californıa Los Angeles 7, California

L. J. PaIge

University of California

Los Angeles 24, California

\section{ASSOCIATE EDITORS}
E. F. BECKENBACH
T. M. CHERRY
D. DERRY

E. HEWITT
A. HORN
L. NACHBIN
M. OHTSUKA

H. L. ROYDEN

M. M. SCHIFFER
E. SPANIER

E. G. STRAUS

F. WOLF

\section{SUPPORTING INSTITUTIONS}

\author{
UNIVERSITY OF BRITISH COLUMBIA \\ CALIFORNIA INSTITUTE OF TECHNOLOGY \\ UNIVERSITY OF CALIFORNIA \\ MONTANA STATE UNIVERSITY \\ UNIVERSITY OF NEVADA \\ NEW MEXICO STATE UNIVERSITY \\ OREGON STATE COLLEGE \\ UNIVERSITY OF OREGON \\ OSAKA UNIVERSITY \\ UNIVERSITY OF SOUTHERN CALIFORNIA
}

\author{
STANFORD UNIVERSITY \\ UNIVERSITY OF TOKYO \\ UNIVERSITY OF UTAH \\ WASHINGTON STATE COLLEGE \\ UNIVERSITY OF WASHINGTON \\ AMERICAN MATHEMATICAL SOCIETY \\ CALIFORNIA RESEARCH CORPORATION \\ HUGHES AIRCRAFT COMPANY \\ SPACE TECHNOLOGY LABORATORIES \\ NAVAL ORDNANCE TEST STATION
}

Mathematical papers intended for publication in the Pacific Journal of Mathematics should be typewritten (double spaced), and the author should keep a complete copy. Manuscripts may be sent to any one of the four editors. All other communications to the editors should be addressed to the managing editor, L. J. Paige at the University of California, Los Angeles 24, California.

50 reprints per author of each article are furnished free of charge; additional copies may be obtained at cost in multiples of 50 .

The Pacific Journal of Mathematics is published quarterly, in March, June, September, and December. The price per volume (4 numbers) is $\$ 12.00$; single issues, $\$ 3.50$. Back numbers are available. Special price to individual faculty members of supporting institutions and to individual members of the American Mathematical Society: $\$ 4.00$ per volume; single issues, $\$ 1.25$.

Subscriptions, orders for back numbers, and changes of address should be sent to Pacific Journal of Mathematics, 2120 Oxford Street, Berkeley 4, California.

Printed at Kokusai Bunken Insatsusha (International Academic Printing Co., Ltd.), No. 6, 2-chome, Fujimi-cho, Chiyoda-ku, Tokyo, Japan.

PUBLISHED BY PACIFIC JOURNAL OF MATHEMATICS, A NON-PROFIT CORPORATION

The Supporting Institutions listed above contribute to the cost of publication of this Journal, but they are not owners or publishers and have no responsibility for its content or policies. 


\section{Pacific Journal of Mathematics}

\section{Vol. 10, No. $2 \quad$ October, 1960}

Maynard G. Arsove, The Paley-Wiener theorem in metric linear spaces ........

Robert (Yisrael) John Aumann, Acceptable points in games of perfect

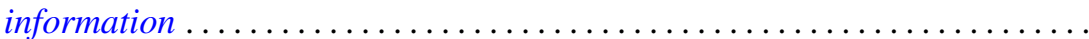

A. V. Balakrishnan, Fractional powers of closed operators and the semigroups generated by them ... . . . . . . . . . . . . . . . . . . . . . . . . . . . . 419

Dallas O. Banks, Bounds for the eigenvalues of some vibrating systems . . . . . 439

Billy Joe Boyer, On the summability of derived Fourier series . . . . . . . . . . . 475

Robert Breusch, An elementary proof of the prime number theorem with

remainder term ...................................

Edward David Callender, Jr., Hölder continuity of $n$-dimensional

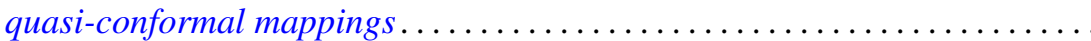

L. Carlitz, Note on Alder's polynomials ......................... 517

P. H. Doyle, III, Unions of cell pairs in $E^{3} \ldots \ldots \ldots \ldots \ldots \ldots \ldots \ldots \ldots \ldots \ldots \ldots . \ldots 21$

James Eells, Jr., A class of smooth bundles over a manifold . . . . . . . . . . . . 525

Shaul Foguel, Computations of the multiplicity function . . . . . . . . . . . . . . 539

James G. Glimm and Richard Vincent Kadison, Unitary operators in

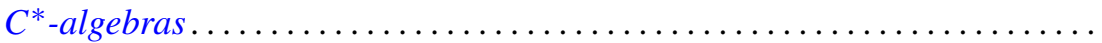

Hugh Gordon, Measure defined by abstract $L_{p}$ spaces . . . . . . . . . . . 557

Robert Clarke James, Separable conjugate spaces ....................

William Elliott Jenner, On non-associative algebras associated with bilinear forms

Harold H. Johnson, Terminating prolongation procedures

John W. Milnor and Edwin Spanier, Two remarks on fiber homotopy type .

Donald Alan Norton, A note on associativity . .

Ronald John Nunke, On the extensions of a torsion module.

Joseph J. Rotman, Mixed modules over valuations rings . . . . .

A. Sade, Théorie des systèmes demosiens de groupoï des . .

Wolfgang M. Schmidt, On normal numbers . .

661

Berthold Schweizer, Abe Sklar and Edward Oakley Thorp, The metrization of

statistical metric spaces

John P. Shanahan, On uniqueness questions for hyperbolic differential

equations

A. H. Stone, Sequences of coverings

Edward Oakley Thorp, Projections onto the subspace of compact operators

L. Bruce Treybig, Concerning certain locally peripherally separable spaces

Milo Wesley Weaver, On the commutativity of a correspondence and a

permutation

David Van Vranken Wend, On the zeros of solutions of some linear complex

differential equations. 